\author{
Attilio Motta* \\ Università degli Studi di Padova
}

\title{
TRADUZIONI E RISCRITTURE: COME LAVORAVA NIEVO (GIORNALISTA)
}

\begin{abstract}
La lingua e le riviste francesi rappresentano per Nievo la mediazione di modelli culturali europei nonché il tramite di vere e proprie fonti testuali a cui il giornalista si rifà con riscritture che sono talvolta ai limiti della traduzione. L'attribuzione a Nievo di un articolo anonimo del Corriere delle Dame del 1859 in base a un pezzo coevo dell'Uomo di Pietra e il reperimento della loro fonte francese su Le Monde illustré forniscono un'occasione unica di confrontare tre testi e entrare nel laboratorio della scrittura per indagare il metodo di lavoro del letterato.

Parole chiave: Nievo giornalista, francese come tramite linguistico e culturale, riviste ottocentesche, riscrittura e traduzione, attribuzione, modus operandi.
\end{abstract}

\section{INTRODUZIONE}

È noto che se le traduzioni nieviane di autori transalpini si limitano ad alcuni testi del quarto libro delle Contemplations di Victor Hugo inseriti nell'ultima delle tre recensioni da lui dedicate all'opera ${ }^{1}$, il ruolo del francese si estende però (con l'eccezione 'classica' di tre testi da Saffo del 1856) a quello di lingua veicolare dell'intero corpus delle altre versioni nieviane (quasi tutte del 1859), di cui Iginio De Luca ha individuato con precisione il tramite (vedi Nievo 1964): Il fiore delle canzoni di Marino Vreto, pubblicato sulla Ricamatrice tra l'aprile e il maggio, deriva infatti dai Contes et Poèmes de la Grèce moderne editi in francese dallo stesso poeta greco (Vreto 1855); le numerose versioni da Heine (l'Intermezzo, Barca e Sveglia dalle Foglie volanti - errore nieviano per "fogli volanti" -, quattro capitoli della Germania e la romanza Un Asra dal Romancero), rimaste inedite vivente Nievo (con l'eccezione del Pellegrinaggio a Kevlaar dai Notturni, pubbli-

*attilio.motta@unipd.it

${ }^{1}$ Cfr. l'articolo Vittore Hugo e il Quarto Libro delle sue Contemplazioni apparso in La Ricamatrice (XI, 17, $1^{\circ}$ settembre 1858, pp. 132-133), ora in Nievo (2008: 119-129). 
cato nel 1859 in una strenna) $)^{2}$, son tutte dipendenti dal volume Poëmes et Légendes par Henri Heine (Parigi, 1855); e le tre canzoni popolari tedesche - anch'esse lasciate manoscritte - erano state già tradotte in francese dallo stesso Heine nell'opera De l'Allemagne (pubblicata nel 1835 me letta da Nievo nell'edizione del '55).

Un intermediario francese ha con ogni probabilità anche l'unica di cui non è identificata la fonte, l'anonima leggenda scozzese Il figlio del settentrione (La Ricamatrice, $1^{\circ}$ maggio 1859), mentre per I doni del Tereck. Ballata russa di Lermontov (16 febbraio dello stesso anno nella medesima rivista) e per la fiaba L'Angelo di Andersen (Giornale delle Famiglie, $1^{\circ}$ marzo 1860, con l'improbabile indicazione «dal danese») Patrizia Zambon sospetta la derivazione dalle traduzioni pubblicate rispettivamente sulle riviste Il Caffè (da Gaetano Branca nel giugno 1855) e Letture di Famiglia (gennaio '60)'.

Unica lingua straniera ben conosciuta da Nievo, il francese svolge più in generale il ruolo di mediatore per alcuni dei modelli culturali, filosofici e letterari più significativi per la sua formazione e le sue opere, non solo transalpini (Rousseau, Stendhal, Vigny, Balzac, George Sand, ma anche Sterne $)^{4}$. Veicolo certo 'minore' di queste influenze fu anche la serie delle riviste francesi che circolavano in Italia al tempo, specie nelle redazioni dei giornali cui Nievo collaborava, e che talora egli cita nell'epistolario, le quali costituirono però anche diretti modelli di tipologia, struttura, temi e toni per la sua attività giornalistica (che conta più di cento testi fra recensioni letterarie e teatrali, articoli satirico-umoristici, saggi, pezzi divulgativi, racconti morali), e talvolta funsero addirittura da vere e proprie fonti testuali rispetto alle quali il trattamento nieviano è ai limiti della traduzione, come dimostrano in particolare tre casi di dipendenza dalla Revue des deux mondes indagati da Sara Garau: il più antico riguarda la sesta parte degli Studii sulla poesia popolare e civile massimamente in Italia, pubblicata su L'Alchimista Friulano del 6 agosto 1854, e dipendente da una riflessione di Charles de Mazade, Jasmin et la poésie populaire méridionale, apparsa sulla Revue il $1^{\circ}$ gennaio del medesimo anno, e taciuta, nonostante una «modalità di ripresa della fonte, che qui come altrove si avvicina alla traduzione»; di due anni dopo, il caso successivo riguarda proprio la prima recensione alle Contemplations di Hugo pubblicata il 24 agosto 1856 sulla Rivista Veneta, in parte dipendente da una di Gustave Planche sulla Revue del 15 maggio dello stesso anno, cui fa questa volta esplicito riferimento; posteriore di quasi altri due anni il terzo episodio, l'articolo satirico Il progresso siamese, edito

\footnotetext{
${ }^{2}$ Cfr. Strenna (1859: 126-128).

${ }^{3}$ Cfr. Nievo 2008: 129-132 (e 393-395), 171-173 (e 402-404).

${ }^{4} \mathrm{Cfr}$. in particolare Garau (2013) e relativa bibliografia.
} 
sul Pungolo del 5 aprile 1858, che ha addirittura due fonti: la Réception de l'ambassade française à Siam di Peyre-Ferry, edita sull'Illustration il 23 maggio del 1857, e Le royaume de Siam et une ambassade anglaise à Bangkok di Charles Lavollée, pubblicato sulla Revue il 15 novembre dello stesso anno ${ }^{5}$.

Ora un nuovo caso viene ad aggiungersi a questa serie, con la particolarità che esso coinvolge non uno, bensì due articoli nieviani, il primo dei quali, sinora attribuito a Nievo solo in forma dubitativa da Patrizia Zambon (Nievo 2008: 64-67, 435-436), viene certificato nella sua paternità proprio dal confronto col secondo: e l'attribuzione è completata dal reperimento della chiara fonte francese di entrambi, come ho già segnalato altrove (cfr. Motta 2018). In questa sede, dopo una sintetica ma necessaria ricapitolazione della vicenda, procederò al confronto fra i tre scritti coinvolti.

\section{UN NUOVO ARTICOLO DI NIEVO}

L'articolo in questione si intitola Il medico delle anime, e apparve anonimo sul Corriere delle Dame dell'8 marzo 1859 (a. LVII, n. 10, p. 78). Eccone il testo:

\section{IL MEDICO DELLE ANIME.}

Si dice che non son belle le cose che non vengono da lontano; ma senza dubbio in fatto di curiosità l'America ci manda le più squisite.

Il dottor Giuseppe Crandon di Charlestewn ${ }^{6}$ osservò che se vi sono i medici del corpo ben vi potrebbero essere anche quelli delle anime. Egli decise di speculare a proprio vantaggio sopra la scoperta di questa nuova professione.

A tale effetto publicò ${ }^{7}$ sopra molti giornali e fece affiggere ai soliti luoghi un avviso il quale notificava al publico che il celebre dottor Giuseppe Crandon prenderà anime in cura ed anche in pensione, e si reca a dar consulti a domicilio per le interessanti e impalpabili malate.

Per sei anni il celebre dottore rimase senza clientele, ma sul settimo fu più avventurato ${ }^{8}$.

${ }^{5}$ Garau (2013: 322, 319-321, 325).

${ }^{6}$ Charlestewn è probabile grafia erronea per Charlestown, nome di almeno cinque città degli Stati Uniti (in Indiana, Maryland, New Hampshire, Rhode Island e Wisconsin), oltre che di un quartiere storico di Boston.

${ }^{7}$ Come il successivo publico, è grafia che riflette lo scempiamento settentrionale, frequente in Nievo come in molte riviste dell'epoca.

8 'fortunato'. 
Un ex-droghiere per nome Hovard ${ }^{9}$, sentendosi probabilmente l'anima malata, ricorse a lui per la guarigione.

Il dottore gli tastò il polso (dell'anima, s'intende), e, dopo matura ponderazione, decise che in un anno di cura lo assicurava guarito.

L'ex-droghiere, avvezzo a contrattare la cannella ed il pepe, volle mercanteggiare anche la salute dell'anima sua; e per quanto il dottore esigesse la mercede di venti dollari, stava fermo a non volerne concedere più di dodici.

S'accordarono, com'era facile a prevedersi, sui quindici, e l'esimio dottore commise l'imprudenza di pattuire che questa somma non gli sarebbe pagata che a guarigione riuscita. Conveniamone che, o il dottore aveva l'anima, cioè la testa, malata più del suo cliente, o che aveva una singolare confidenza nell'arte sua.

Il fatto sta che la cura incominciò, e se noi non ne diamo i particolari, gli è soltanto perchè i giornali americani passano sotto silenzio questo periodo importantissimo del loro racconto.

Dopo l'anno pattuito, il dottore pretese da Hovard i quindici dollari, e questi si rifiutò di pagarglieli col pretesto che la guarigione era tutt'altro che riescita ${ }^{10}$, Pretendi di là, nega di qua, non c'era altro modo di conciliazione che andare ai tribunali.

Infatti la petizione fu intimata, la difesa fu opposta, e undici membri ${ }^{11}$ del giurì sopra dodici sentenziarono che Hovard non dovesse pagare i quindici dollari a Crandon, non sentendosi egli l'anima perfettamente guarita. Il duodecimo giudice si astenne dicendo di dubitare fortemente che il citato Hovard avesse un'anima.

In America le sentenze devono adottarsi all'unanimità, per cui l'affare fu rimessa ${ }^{12}$ ad una seconda seduta.

Crandon oppose allora che probabilmente l'anima di Hovard era incurabile, se in un anno di assidue cure non era guarita. Ma Hovard rimbeccò che l'anima sua era tanto poco incurabile che se a metà della cura il dottore non si fosse capricciosamente assentato per assistere ad un meeting, egli era certo che sarebbe risanata completamente. Ma la malaugurata partenza del medico le avea cagionato una ricaduta pur troppo irreparabile.

Questa volta il giurì fu unanime; giudicò pazzi tutt'e due i contendenti, e mandò il medico ed il malato in piazza a far ridere i monelli. Anche noi siamo dell'avviso di quest'ultimo giurì ma se questa professione sognata dal dottor Crandon fosse possibile, confessiamolo che sarebbe anche in Europa d'un'urgente opportunità e d'un vantaggio immediato. Opineremmo che una facoltà $a d$ hoc si instituisse in ogni università, che rimanessero aboliti per sempre gli sbadigli, i sospiri, e sopratutto le lamentazioni poetiche delle anime malate.

${ }^{9}$ Altra grafia erronea per Howard (come nell'articolo "gemello"); si noti l'uso della preposizione per, che appare un (iper)francesismo ('par nom').

${ }^{10} \mathrm{E}$ forma toscaneggiante che alterna, al tempo e in Nievo, con riuscita (cfr. Mengaldo 1987: 51).

${ }^{11}$ Si noti la consueta preferenza nieviana per il numero undici (accompagnata nel pezzo a quella per un altro numero primo, il sette).

${ }^{12} \mathrm{Si}$ tratta di un notevole francesismo, in quanto il participio passato è accordato ad affare femminile (come il francese affaire). 
Come accennato, la paternità nieviana del pezzo è certificata, oltre che da indizi minori, dalla sua sostanziale sovrapponibilità con l'articolo Per le anime malate, pubblicato un mese dopo, il 9 aprile 1859, sull'Uomo di Pietra (a. III, n. 15: d'ora in poi UP), a firma "Todero", pseudonimo la cui riconducibilità a Nievo è assolutamente certa ${ }^{13}$ :

No, no, no, no! non parlo con voi!

Appena strombettato il titolo, mi veggo venir intorno una calca confusa di zitellone ammuffite, di droghieri falliti, di commessi di studio in disponibilità ${ }^{14}$, di poetucoli sbagliati, e di primi amorosi tisici!

Sbagliate uscio, carini miei! Voi non avete malata l'anima ma il corpo. Picchiate dal dottor fisico qui presso che vi darà un consulto: io mi rivolgo unicamente e specialmente a quei disgraziati che hanno proprio l'anima malata come l'ho malata io.

Non è il pizzicor ${ }^{15}$ dei sensi, non lo stomaco vuoto, nè il bisogno di toeletta che mi fa strillare. È proprio una malattia dell'anima, vera come un callo, ed assoluta come una prima donna. Del resto questi casi disperati non sono rari nel secolo del Werther di Goethe e del Manfredo di Byron ${ }^{16}$, ed è per questo appunto ch'io mi affrettai a scrivere un articolo per dare al colto pubblico e specialmente a voi, anime malate, la buona, la buonissima novella.

Il dottor Alberto Crandon di Nuova Yorch, spiritualista di professione, osservò che se le dotte e pietose cure d'una porzione preclarissima ${ }^{17}$ dell'umana famiglia si dedicano a guarire i corpi malati dei loro simili, analoghe cure dovrebbero prestarsi a coloro che, sani di corpo, hanno pur l'anima affetta da qualche malattia.

Detto, fatto, si sfregolò le mani ed appese fuori della propria finestra il seguente cartello:

Alberto Crandon, dottore spiritualista, cura e guarisce le affezioni morali sì croniche che acute: fa visite a domicilio e prende anime in pensione: assume cure a cottimo e fa abbonamenti: il tutto a prezzi discretissimi.

${ }^{13}$ Per l'articolo cfr. Nievo (2015: 678-683). L'attribuzione dello pseudonimo è garantita da una dichiarazione dello stesso Nievo nella lettera alla madre del 21 febbraio 1858: «Hai ricevuto i giornali dallo Zio Augusto? Sull’ Uom di Pietra le corrispondenze da Nizza e Venezia e gli articoli firmati SSSSS ed Arsenico son miei. Ti prego a non perder quei giornali» (vedi Nievo 1981: 479).

${ }^{14}$ «stato d'impiegato civile o di milite, che rimandisi a tempo dal servigio che prestava, con parte di stipendio, o tutto, potendo essere quandochessia richiamato»: così il Dizionario Tommaseo-Bellini (1861-1879, d'ora in poi TB), peraltro critico sull'uso del termine, cui pone accanto due croci, e della locuzione, a cui preferirebbe a disposizione; oggi si direbbe 'in mobilità'.

15 'prurito', 'smania' (è dantismo, Inf. xxIx, 81).

${ }^{16}$ Il poema drammatico in tre atti Manfred (1817), di forte impronta romantica, mette in scena il senso di colpa del protagonista per un inconfessabile peccato.

17 'illustrissima' (lett.); il doppio superlativo rientra nel gusto nieviano per l'accumulo delle alterazioni. 
Il cartello dell'audace novatore ${ }^{18}$ stette appeso alla sua finestra due anni, senzachè un uomo bussasse alla porta colla premeditata intenzione di farglisi cliente.

Allora il pratico spiritualista prese la risoluzione di aggiungere: a prezzi discretissimi e posticipati.

Quest'ultima parola scritta a grandi caratteri fermò l'attenzione del signor Giorgio Hovard, onesto ex-droghiere che si accorgeva da qualche tempo di aver l'anima gravemente ammalata.

Risalendo dall'ultima parola del cartello alla prima capì che era il suo caso e si precipitò nella stanza del dottor Crandon. Incontro commoventissimo! Come di due pallottoline di mercurio che si fossero corse dietro per tutta la tavola, prima di amalgamarsi.

Il dottore tastò il polso all'anima del signor Hovard, capì di che si trattava, e giudicò necessario un anno di cura.

Un anno! corbezzoli, non è poco! Ma trattandosi che per quaranta franchi il dottore in capo a quel termine guarentiva ${ }^{19}$ la completa guarigione, Hovard si rassegnò.

Non narreremo gli episodii, i particolari e le vicissitudini della cura che fu continuata e condotta a termine d'ambe le parti con rara costanza. Dico soltanto che in capo all'anno fissato il dottore fu preciso a pretendere i quaranta franchi affermando già susseguita in merito ${ }^{20}$ delle sue prescrizioni la guarigione dell'anima del signor Hovard.

Hovard rifiutò affermando dal canto suo che l'interessante ammalata anzichè essersi pienamente ristabilita avea da ultimo peggiorato in modo spaventevole. Crandon trattò questa ragione come un pretesto trovato per negare la somma e ricorse al Tribunale.

La perizia sull'anima del signor Hovard diventava indispensabile, ed essendo un caso non previsto dai codici americani, lasciarono che sentenziasse la scienza infusa dei signori giurati.

Il consesso era composto di dodici membri, numero poco prediletto dagli studiosi della cabala ${ }^{21}$. Essi udirono le ragioni e le obbiezioni, le prove e le eccezioni, il fatto e la negativa ${ }^{22}$, il pro' ed il contro. Ascoltarono con raccapriccio la irreparabile ricaduta dell'anima rea convenuta quando un capriccio del medico curante che avea voluto assistere ad uno spettacolo di corse l'avea lasciata per tre giorni in balìa di sè stessa.

$\mathrm{Ah}$, sconsigliato dottore Crandon ${ }^{23}$ ! gli è così dunque che voi mettete a frutto le vostre filantropiche invenzioni!? Chi vi ha insegnato a cominciar le cose ed a non

18 'innovatore' (è forma ottocentesca).

${ }^{19}$ Forma concorrente di garantiva. Si noti il 'che' polivalente nel precedente trattandosi che.

20 'conseguita per merito' (con raro uso trans. e sign. non attestato dai dizionari, che si limitano a 'seguire').

${ }^{21}$ Cfr. supra, nota 15.

22 'la negazione' (TB).

23 'sconsiderato', 'che agisce senza riflessione'; si noti l'assenza di apocope in dottore, tratto diffuso in Nievo. 
terminarle? a far il civettone ${ }^{24}$ colle anime, ed a piantarle sul più bello? a principiare un miracolo ed interromperlo per un steeplechaise ${ }^{25} \ldots$

Undici giurati decisero che poichè l'anima rea convenuta sfuggiva alla perizia dei tecnici americani, il suo legittimo possessore soltanto poteva farsi giudice e garante della guarigione, e che siccome il possessore suddetto, l'infelice ex-droghiere Hovard, affermava per giuramento esser dessa ${ }^{26}$ ridotta a peggiore stato di prima, così il dottor Crandon non aveva attenuto i patti stabiliti e non poteva pretendere neppur un soldo della somma pattuita.

Il duodecimo giurato che aveva letto Voltaire giudicò a parte che riteneva invalido e nullo il contratto perchè era assai dubbio che l'infelice droghiere Hovard possedesse un'anima.

Per quel giorno fu nullo il giudizio, giacchè in America non si decide che all'unanimità, ma la cosa ha fatto chiasso e sorsero a migliaja gli uomini e le donne patetiche che pretesero di aver un'anima e di sentirla malata. Il dottore Crandon soppresse dal suo cartello quella brutta parola posticipato ed è sulla via di diventar milionario come il benemerito Pagliano ${ }^{27}$ : - Oh nobilissimo esempio!

Animato da filantropico successo, io mi professo ${ }^{28}$ tosto di impartire all'Europa i benefizii della scoperta americana.

Ebbi dal dottor Crandon e da altri distinti luminari della nuova scienza la migliore ricetta. Siccome peraltro il progresso d'una dottrina qualunque deve esser continuo, così io ho pensato di riprenderla al punto dove l'illustre inventore l'ha condotta, e di non tornare ai primi passi del pagamento posticipato.

All'usanza dei pronti contanti, mi trovo eccitato ${ }^{29}$ anche dalla necessità di guarire l'anima mia in certe sue piaghe abituali.

Come? direte voi - guarir l'anima coi denari? lo spirito colla materia?

Eh, signori miei, e chi conosce appuntino ${ }^{30}$ gli intimi e misteriosi legami che uniscono lo spirito alla materia? Io solo, unico depositario della nuova scienza in Europa, ho diritto di conoscerli.

Non espongo dunque nessun cartello; ma fin d'ora mi metto a disposizione del pubblico pel canale cortese dell'Uomo di Pietra.

A pagamento anticipato guarentisco la guarigione anche delle anime tisiche in ultimo grado. La ricetta è infallibile. Soltanto prevengo i signori malati che chi

${ }^{24}$ «per dispr. d'Uomo che con più vanità e malizia che garbo civetta con femmine» (TB).

${ }^{25}$ Grafia erronea (e francesizzante?) per steeplechase, 'corsa a ostacoli' (ingl.)

26 'essa': aulicismo che mima qui il formalismo del linguaggio giuridico, come il successivo non aveva attenuto i patti, con uso transitivo (attestato però come comune da TB) del verbo solitamente riflessivo.

${ }^{27}$ Il baritono e farmacista Girolamo Pagliano (1801-81), inventore di un fortunatissimo sciroppo purgativo che veniva venduto avvolto nel libretto La medicina dei padri di famiglia (Firenze, Le Monnier, 1844), vero e proprio best seller della metà del secolo.

28 'proclamo', con uso del rifl. a reggere una prop. sub. che non è attestato dai dizionari.

${ }^{29}$ All'usanza... eccitato vale 'sollecitato all'uso'.

30 'minuziosamente'. 
pretende la restituzione del pagamento per fallita guarigione, ha a suo carico la prova dell'esistenza non solo, ma anche della malattia dell'anima sua.

Si sa il numero degli abitanti del globo nel secolo XIX: ma quello delle anime? - La statistica non se ne cura. Lo sapranno e lo diranno i posteri!

TODERO.

Appare evidente come il nucleo narrativo del pezzo sia il medesimo del Medico delle anime: la circostanza e la contiguità temporale tra le due pubblicazioni sono peraltro assolutamente in linea con altri casi in cui Nievo riutilizza i medesimi materiali per articoli destinati a riviste diverse; nel caso in questione, inoltre, la precedenza del Medico delle anime appare non solo editoriale, ma, come vedremo meglio tra poco, anche compositiva: il raccontino infatti è più lineare e meno ricamato rispetto all'articolo di $U P$, il cui titolo peraltro riprende proprio il sintagma finale del primo.

\section{LA FONTE FRANCESE}

Un'attenta analisi linguistica del Medico delle anime evidenzia però almeno un paio di indizi fortemente francesizzanti, tanto da aver fatto sospettare a chi scrive che essi potessero essere non il frutto di un'oltranza attiva di Nievo, ma di una sua inerzia traduttoria; d'altra parte la presenza nel pezzo di nomi propri ha reso più facile la ricerca con gli strumenti informatici, tanto che la biblioteca digitale Gallica ha consentito di individuare senza ombra di dubbio la fonte nella parte finale dell'articolo Courrier du palais, pubblicato su Le Monde illustré (sottotitolo «Journal Hebdomadaire») del 29 gennaio 1859 (III, 94, p. 78), a firma Petit-Jean.

La rivista, nata nell'aprile del 1857, era un settimanale d'attualità costituito di 16 pagine in folio dal costo di 30 centesimi, aveva sede presso la Librairie Nouvelle di Boulevard des Italianes 15, e, secondo lo studioso francese Jean-Pierre Bacot (2005: 76-78), era edita all'epoca dal proprietario di questa, ossia il semisconosciuto Achille Bourdilliat, già direttore del Moniteur universel, anche se Gallica riporta come suo direttore lo scrittore di origini spagnole Charles Yriarte; di orientamento filoimperiale, essa era ampiamente occupata da illustrazioni («gravures») per lo più non originali, ma recuperate dai tabloid inglesi, particolare che se non altro segnala come una certa nonchalance nel riuso dei materiali editoriali non fosse ad esclusivo appannaggio delle riviste italiane o di Nievo, come si ricava peraltro indirettamente dall'avvertenza, posta tra le informazioni sotto la testata, che «La reproduction et la traduction sont interdites».

Più complicato capire a chi corrisponda il nome dell'autore Petit-Jean, se si tratti di un nom de plume o di un cognome, nel qual caso si aprirebbero diverse possibilità. Non ci addentreremo tuttavia nella questione, che non 
è centrale per i nostri fini, in quanto è piuttosto evidente come l'attenzione nieviana per il modello non derivi dall'autore, ma dalla gustosità dell'aneddoto riportato, che si disponeva naturalmente ad essere utilizzato ai suoi scopi, in direzioni pure apparentemente contraddittorie, e cioè da un lato nel senso di una sua riproposizione narrativa tendenzialmente fedele a fini di intrattenimento, e dall'altro nella sua parziale deformazione e nel suo progressivo adattamento alla natura umoristico-satirica, ma anche politica, del giornalismo nieviano maggiore ${ }^{31}$.

L'articolo de Le Monde illustré tratta di ambienti giudiziari, e in particolare sembra prendere di mira l'abuso del ricorso ai tribunali per questioni di piccola entità o relative comunque alla sfera letteraria e culturale. Più nel dettaglio esso inizia citando il drammaturgo vaudeville e librettista parigino Eugène Scribe (1791-1861) e i personaggi della sua pièce La camaraderie ou la Courte échelle (Il cameratismo, 1837), in particolare l'avvocato spiantato de Varennes, pretendente la mano della nobile Agathe de Miremont, nonostante l'opposizione del padre di lei. Prosegue parlando poi di una accusa di plagio a Théodore Barrière, altro commediografo parigino (1823-78), famoso per Le feu au couvent (Fuoco al Convento, 1859), ma qui citato per la precedente Cendrillon (Cenerentola, 1858), che era stata additata per essere stata ripresa da un testo tedesco. Per dimostrare l'assurdità di risolvere dispute letterarie con carte bollate, l'articolista ricorda poi il letterato Edmond About (1828-85), il quale aveva risposto all'accusa che il suo romanzo Tolla (1855) avesse modelli italiani con la successiva produzione $^{32}$, e Charles-Guillaume Étienne, attaccato per l'opera Les deux gendres (1810); diverso - prosegue Petit-Jean - se l'offesa riguarda la vita privata, come accaduto al pianista ginevrino Sigismund Thalberg (1812-71), tirato in ballo in una storia di prostituzione parigina quando in realtà si era già trasferito a Napoli; il redattore racconta quindi un aneddoto relativo alla lite tra lo strillone del Figaro-Programme e il direttore del teatro Délassements-Comiques, Sari, condannato a risarcire il primo, che aveva cacciato a calci nel sedere, ospitandolo all'interno dello stabile.

È a questo punto che l'articolista invita a gustare quella che definisce una «friandise judiciaire» (ossia una "chicca giudiziaria") che arriva «en ligne droite» dagli Stati Uniti, e inizia a raccontare la storia che fa da modello a Nievo:

${ }^{31}$ Che è quanto accade anche nel caso del Progresso siamese rispetto al diverso orientamento politico delle sue fonti (vedi Garau 2013: 325).

${ }^{32}$ Secondo Garau (2013: 318n), alle «ciancie di About» Nievo fa polemico riferimento nella Corrispondenza da Venezia del 2 ottobre 1858 (UP, II, 40: cfr. Nievo 2015: 577-578), con probabile allusione proprio al romanzo Tolla, «di ambientazione romana», pubblicato a puntate sulla Revue des deux Mondes nel 1855. 
$[\ldots]$

Veuillez bien maintenant déguster, comme il convient, cette friandise judiciaire qui nous arrive en ligne droite des États-Unis d'Amérique.

Joseph Crandon, citoyen de l'État du Maine ${ }^{33}$, se sentait l'âme malade; le vice l'envahissait, que c'en était pitié. Il alla trouver le révérend Hiram Osgood, un médecin d'âmes de ce pays-là, qui passe pour un homme habile et opérant dans les prix doux. Le docteur hocha la tête: l'âme de Joseph Crandon lui paraissait diablement avariée; néanmoins il consentit à en entreprendre la guérison. Pour prix de ses soins, il demanda quinze dollars, - et ces conventions furent constatées par un acte en due forme.

Quinze dollars pour nettoyer l'âme de Joseph Crandon! y aviez-vous bien pensé, honnête Osgood, quand vous avez entrepris ce travail surhumain!

Au bout d'une année, le docteur était à bout d'efforts et de peines; mais l'âme de Crandon ne s'en portait pas mieux, et la preuve, c'est que lorsque le docteur a réclamé ses quinze dollars, Crandon les lui a carrément refusés: «Pas de guérison, pas de salaire, a dit Crandon; guérissez-moi ou laissez-moi tranquille!»

Le révérend Osgood, qui ne soigne pas les âmes pour l'amour de Dieu, a, en vertu de son acte, assigné son malade devant la cour de Columbia!

- Eh quoi! s'écrie-t-il, j'aurai épuisé sur cette âme corrompue tout mon temps et toute ma science, j'aurai prêché et reprêché ce malheureux ad sudorem, et, sous prétexte qu'il n'est pas guéri, il me refusera mon salaire! Hercule a nettoyé les écuries d'Augias, mais Hercule était un demi dieu: moi qui ne suis qu'un simple mortel, puis-je être tenu à l'impossible?

- Comparaison aussi fausse qu'impertinente, répond le citoyen Crandon! Mon âme était malade, c'est vrai; souillée d'ordures, c'est vrai; mais non pas incurable. Un peu de soin aurait suffi pour la purger de son mal. Déjà elle était en train d'être sauvée quand le docteur l'a quittée brusquement pour s'en aller, dans un meeting de Jonesport ${ }^{34}$, jouer à l'homme politique: elle a alors éprouvé une rechute et voilà comment elle se trouve aujourd'hui plus éloignée de la guérison qu'auparavant. En résumé, damné pour damné, je préfère l'être avec quinze dollars de plus dans ma poche.

Sur douze jurés, onze ont opiné en faveur du docteur. Le douzième a émis un vote contraire «attendu, a-t-il dit, qu'il ne paraissait pas démontré que Crandon eût une âme à sauver». La faim - ce moyen de persuasion à l'usage des jurys anglais et américains - a été impuissante à concilier ces divergences et à amener un verdict unanime. L'affaire a été renvoyée à une prochaine session.

$\mathrm{Si} \mathrm{jamais} \mathrm{vous} \mathrm{trouvez} \mathrm{un} \mathrm{procès} \mathrm{plus} \mathrm{joli} \mathrm{que} \mathrm{celui-là,} \mathrm{je} \mathrm{consens} \mathrm{à} \mathrm{perdre} \mathrm{mon}$ nom de...

PETIT-JEAN.

${ }^{33}$ Il cognome è attestato in New England, e uno Joseph Crandon «son of Philip Crandon [...], was born in Fairhaven, July 21, 1802, and died August 21, 1883» (Cutter 1908: 1869).

${ }^{34}$ Piccola località marina del Maine, con poco più di un migliaio di abitanti (allora e oggi). 
L'identificazione della fonte appare certa, e sebbene a rigore non sia impossibile immaginare un'ulteriore intermediazione per il tramite di una delle tante riviste (francesi o italiane) del tempo, tale evenienza appare scongiurata dai passaggi più fedeli (calchi, etc.) e anche dalla natura "nieviana" delle modifiche.

D'altra parte se il reperimento del modello estende quantitativamente e cronologicamente la casistica di un modus operandi nieviano, tanto da far valutare opportuna un'indagine più sistematica alla ricerca di ulteriori episodi del genere, esso ha anche una sua eccezionalità in quanto la derivazione dalla fonte non di uno, ma di ben due pezzi nieviani, tra loro vicini nel tempo ma non privi di differenze, ci mette nella condizione più unica che rara di entrare nel laboratorio redazionale dell'autore e verificare il suo metodo di lavoro in relazione alla differente destinazione editoriale e agli scopi ad essa connessi.

\section{IL CONFRONTO FRA I TESTI}

Una prima considerazione, puramente quantitativa, riguarda la diversa estensione dei tre pezzi: la fonte francese conta infatti, nella porzione interessata, 2642 caratteri; la prima versione nieviana, quella di $C D$, arriva a 3382, mentre la seconda, quella di $U P$, è quasi raddoppiata rispetto alla precedente: primo segno evidente di come la modalità "ciarliera" adottata per il principale giornale umoristico-satirico milanese del tempo spingesse l'autore a una revisione più massiccia della struttura e del tono dell'aneddoto su cui inizialmente aveva effettuato interventi minori seppur non privi di interesse. Per rendere praticabile un confronto fra $i$ tre testi che metta in evidenza le differenze narrative e strutturali, ho costruito una tabella che ne riporta le sequenze essenziali:

\begin{tabular}{|l|l|l|}
\hline \multicolumn{1}{|c|}{$\begin{array}{c}\text { (a) COURRIER } \\
\text { DU PALAIS }\end{array}$} & \multicolumn{1}{|c|}{$\begin{array}{c}\text { (b) IL MEDICO DELLE } \\
\text { ANIME }\end{array}$} & \multicolumn{1}{c|}{$\begin{array}{c}\text { (c) PER LE ANIME } \\
\text { MALATE }\end{array}$} \\
\hline $\begin{array}{l}\text { Breve introduzione su aned- } \\
\text { doto gustoso }\end{array}$ & $\begin{array}{l}\text { Breve introduzione su aned- } \\
\text { doto gustoso }\end{array}$ & $\begin{array}{l}\text { Ampia e satirica introduzione } \\
\text { sui destinatari dell'aneddoto }\end{array}$ \\
\hline $\begin{array}{l}\text { Il cittadino del Maine Joseph } \\
\text { Crandon avverte la propria } \\
\text { anima malata }\end{array}$ & $\begin{array}{l}\text { Il dottor Giuseppe Crandon } \\
\text { di Charlestewn pensa che se } \\
\text { ci sono i medici del corpo ci } \\
\text { possono essere anche quelli } \\
\text { delle anime }\end{array}$ & $\begin{array}{l}\text { Il dottor Alberto Crandon di NY, } \\
\text { spiritualista, pensa che se ci sono } \\
\text { i medici del corpo ci possono } \\
\text { essere anche quelli delle anime }\end{array}$ \\
\hline $\begin{array}{l}\text { e si rivolge al reverendo } \\
\text { Osgood, locale "medico delle } \\
\text { anime" }\end{array}$ & $\begin{array}{l}\text { Decide quindi di offrire i i } \\
\text { suoi servigi e pubblica un } \\
\text { annuncio }\end{array}$ & $\begin{array}{l}\text { Decide quindi di offrire i suoi } \\
\text { servigi e pubblica un annuncio }\end{array}$ \\
\hline
\end{tabular}




\begin{tabular}{|c|c|c|}
\hline & & $\begin{array}{l}\text { Dopo due anni, non essendosi } \\
\text { presentato nessuno, aggiunge } \\
\text { all'annuncio "a prezzi discre- } \\
\text { tissimi e posticipati" }\end{array}$ \\
\hline & $\begin{array}{l}\text { Al settimo anno l'ex dro- } \\
\text { ghiere Howard si rivolge } \\
\text { a lui, }\end{array}$ & $\begin{array}{l}\text { Quest'ultima parola attrae l'at- } \\
\text { tenzione dell'ex droghiere Gior- } \\
\text { gio Hovard, che va dal dottore. }\end{array}$ \\
\hline & $\begin{array}{l}\text { Crandon lo visita e gli as- } \\
\text { sicura la guarigione in un } \\
\text { anno }\end{array}$ & $\begin{array}{l}\text { Crandon lo visita e gli assicura } \\
\text { la guarigione in un anno }\end{array}$ \\
\hline $\begin{array}{l}\text { Questi lo prende in cura per } \\
15 \text { dollari e gli fa firmare un } \\
\text { contratto. }\end{array}$ & $\begin{array}{l}\text { Dopo un mercanteggiamento } \\
\text { i due pattuiscono un com- } \\
\text { penso di } 15 \text { dollari a guari- } \\
\text { gione avvenuta. }\end{array}$ & $\begin{array}{l}\text { Hovard accetta la terapia per } \\
40 \text { franchi. }\end{array}$ \\
\hline $\begin{array}{l}\text { Dopo un anno, Crandon non } \\
\text { è guarito ma il reverendo pre- } \\
\text { tende il compenso e lo porta } \\
\text { in tribunale. }\end{array}$ & $\begin{array}{l}\text { Dopo un anno Crandon pre- } \\
\text { tende il compenso, Hovard } \\
\text { sostiene di non esser guarito, } \\
\text { si va in tribunale. }\end{array}$ & $\begin{array}{l}\text { Dopo un anno Crandon dichiara } \\
\text { l'anima guarita e pretende il } \\
\text { compenso, ma Hovard sostiene } \\
\text { di star peggio: si va in tribu- } \\
\text { nale }\end{array}$ \\
\hline $\begin{array}{l}\text { Il reverendo sostiene Crandon } \\
\text { incurabile, questi accusa l'al- } \\
\text { tro di aver interrotto la cura re- } \\
\text { candosi ad un meeting politico } \\
\text { a Jonesport (Maine). }\end{array}$ & & $\begin{array}{l}\text { Ci vorrebbe la perizia, si lascia } \\
\text { la parola ai } 12 \text { giurati, che } \\
\text { ascoltano ragioni e obiezioni } \\
\text { e apprendono della ricaduta } \\
\text { dell'anima per un'assenza del } \\
\text { medico recatosi a uno spettacolo } \\
\text { di corse. Commento ironico del } \\
\text { narratore sulla sua imperizia. }\end{array}$ \\
\hline \multirow[t]{4}{*}{$\begin{array}{l}11 \text { giudici danno ragione al me- } \\
\text { dico, il } 12^{\circ} \text { vota contro perché } \\
\text { non si sa se Crandon ha un'ani- } \\
\text { ma: si rinvia a nuovo giudizio. }\end{array}$} & $\begin{array}{l}11 \text { giudici danno ragione ad } \\
\text { Hovard, ma il } 12^{\circ} \text { si astie- } \\
\text { ne dubitando che egli abbia } \\
\text { un'anima: per le regole ameri- } \\
\text { cane si va a nuovo giudizio. }\end{array}$ & $\begin{array}{l}11 \text { giurati danno ragione ad Ho- } \\
\text { vard, ma il } 12^{\circ} \text { dubita che egli } \\
\text { abbia un'anima: per le regole } \\
\text { americane il giudizio è nullo. }\end{array}$ \\
\hline & $\begin{array}{l}\text { Crandon sostiene Hovard } \\
\text { incurabile, questi accusa il } \\
\text { medico di aver interrotto la } \\
\text { cura per un meeting. }\end{array}$ & \\
\hline & $\begin{array}{l}\text { Il giurì dichiara pazzi en- } \\
\text { trambi. }\end{array}$ & $\begin{array}{l}\text { Il processo ha attirato l'attenzio- } \\
\text { ne, Crandon ha tolto "posticipa- } \\
\text { to" e ha fatto soldi. }\end{array}$ \\
\hline & $\begin{array}{l}\text { Se la professione esistesse } \\
\text { sarebbe utile in Europa per } \\
\text { eliminare gli sbadigli. }\end{array}$ & $\begin{array}{l}\text { Il redattore professa di voler } \\
\text { importare la pratica in Europa, } \\
\text { ristabilendo il pagamento anti- } \\
\text { cipato, ma avverte che in caso } \\
\text { di mancata guarigione sarà } \\
\text { necessario dimostrare di avere } \\
\text { l'anima per essere risarciti. }\end{array}$ \\
\hline
\end{tabular}


Per dar corso ordinato al doppio confronto, analizzeremo prima le differenze tra (b) e (a), quindi quelle tra (c) e (b). Nella prima versione Nievo rispetta tendenzialmente la struttura agile dell'aneddoto, come dimostra il fatto che esso è introdotto da una frase sostanzialmente sovrapponibile alla fonte, ma ciò non significa che egli non intervenga in maniera significativa sui presupposti stessi della storia, con una serie di modifiche:

1. La prima è quella strutturale di mutamento di ruoli e personaggi che si concretizza anche in una traslazione di nome e nella scelta del titolo: la funzione svolta dal reverendo Osgood, infatti, passa a un medico, chiamato Crandon, che nell'aneddoto francese era il nome del paziente, mentre questi diviene l'ex droghiere Howard; nel passaggio dalla coppia reverendo/cittadino a quella medico/commerciante avvengono dunque due mutamenti importanti: da un lato il ruolo religioso, forse ritenuto insieme ingombrante e limitante le possibilità satiriche, viene sostituito con quello medico, che calamiterà tutta l'ironia del pezzo e, coerentemente, ne "conquista" il titolo: scelta sulla quale può aver avuto un ruolo il modello del romanzo Stello ou le diable bleu di Alfred de Vigny (1832), capostipite della tradizione dei "medici delle anime", che, tradotto nel 1835, Nievo aveva letto in gioventù, traendone forse anche delle suggestioni per l'Antiafrodisiaco ${ }^{35}$. Dall'altro la specificazione "commerciale" del paziente apre al pezzo anche la dimensione moralistica sulla società borghese americana, la cui individuazione vuole essere forse più evidente nella precisazione geografica di una città (Charlestown) rispetto a uno stato (Maine), e che è presente in altre opere di Nievo, dal Barone di Nicastro (in cui compare anche il motivo giudiziario) all'articolo Gli ultimi amanti delle illusioni, del $1860^{36}$.

2. Coerentemente a queste premesse, l'iniziativa passa dal paziente al medico, che Nievo caratterizza con finalità "venali", attribuendogli un sillogismo assente nel modello ("poiché esistono i medici del corpo, allora possono esserci anche quelli delle anime") e la pubblicazione dell'annuncio

${ }^{35}$ Cfr. Nievo 1981: 136 (19.7.50): «Voglio darti a leggere un romanzo un po’ umoristico, $[\ldots]$ I diavoli turchini; e ti assicuro che se i diavoli turchini sono tanto spaventosi, non trovo nella mia immaginazione un genio tanto orribile che possa dipingermi i loro fratelli, color di fuliggine»; Fausta Samaritani scrive che il volume, A. Di Vigny, Stello o i diavoli turchini. Prima versione italiana di Luigi Masieri, Milano, Truffi, 1835, compariva nella biblioteca di Nievo, legato «da un'unica copertina» insieme al Viaggio sentimentale di Sterne «tradotto da Didimo Chierico (Ugo Foscolo), nell'edizione milanese del 1933» (Samaritani \& Zambon 2002: 56). Per le possibili influenze sulle opere nieviane, cfr. Gorra 1985: 582 ss. e Chaarani Lesourd 2002.

${ }^{36}$ Cfr. Ippolito Nievo, Il Barone di Nicastro (in Nievo 1967), in part. il cap. XVI, in cui il protagonista viene arrestato appena sbarcato a New York, e subisce un grottesco processo da cui si salva con il pagamento di una multa; e Arsenico, Gli ultimi amanti delle illusioni, UP, IV, 9, 21 gennaio 1860, in Nievo (2015: 698-706). 
con la chiara intenzione di far soldi. In linea con questo indirizzo, Nievo inserisce inoltre il mercanteggiamento sul prezzo della terapia, con evidente intento di satira del materialismo americano.

3. Altra importante innovazione strutturale è la duplicazione del processo: nel modello infatti il rinvio a un nuovo giudizio lascia spazio a un finale aperto, mentre Nievo anticipa la sentenza non unanime, che, seppur non particolarmente motivata, gli consente di rinviare lo scambio di accuse al secondo processo, il quale non è semplicemente annunciato, ma svolto. Il raccontino ne deriva una maggiore complicazione, ma guadagna un finale umoristico con la seconda sentenza, che dichiara pazze entrambe le parti.

4. Questa tendenza all'articolazione è tanto più significativa in quanto procede di pari passo a un fenomeno apparentemente contrario, e cioè l'eliminazione dei discorsi diretti dei personaggi e della teatralizzazione dibattimentale: in Nievo infatti il racconto vince sul discorso, e la sintesi della voce narrante sulla plurivocità della fonte.

5. Inoltre, se Nievo non aveva toccato l'introduzione, all'aneddoto aggiunge però una conclusione contestualizzante, con il riferimento all'utilità che la professione avrebbe per eliminare gli sbadigli in un'Europa che è evidentemente in preda ad una crisi di sonno. Essa contiene una chiara allusività politica che nel metodo non è diversa da quella di una parte significativa della sua produzione giornalistica, e nel merito rimanda alla lunga attesa, propria del "decennio di preparazione", di qualcosa che smuova l'equilibrio politico rimettendo in moto il processo unitario nazionale.

Le tendenze accennate nella prima rielaborazione sono ancora più evidenti nella seconda, da (b) a (c), sebbene con alcuni riavvicinamenti ad (a):

6. Innanzitutto cambia il titolo (scelta che ha però anche motivi editoriali: due articoli con lo stesso titolo, seppur su giornali diversi, avrebbero potuto essere notati: e infatti Nievo fa così tutte le volte che si trova a 'riciclare' un suo pezzo); di (b) Nievo conserva la parola anime, ma sposta l'attenzione dal medico alla malattia, recuperando così, come in un singolare domino da coblas capfinidas (o da «lascia e prendi») la frase finale dell'articolo precedente.

7. A questo collegata, sebbene decisamente meno obbligata, è la modifica successiva: infatti il brevissimo prologo di (a) e (b) viene sostituito da una lunga introduzione umoristica che, partendo appunto dal titolo, instaura la modalità allocutoria assente nei modelli - se si eccettua l'ultimo capoverso di (a) - e tanto cara invece al Nievo giornalista maggiore (nonché parte essenziale dell'effetto colloquiale che caratterizza la sua prosa). Nella nuova introduzione trovano posto tanto la caricatura di tipi umani, con relativa deformazione linguistica (zitellone, poetucoli, ma anche, autoironicamente, strombettato), quanto una vena lievemente malinconica, troppo esile per 
autorizzare interpretazioni autobiografiche, ma non estranea ad altri scritti nieviani, e non incompatibile, alla luce di quanto si vedrà poi, con una lettura politicamente allusiva.

8. Assistiamo poi a un nuovo cambio della città (da Charlestewn alla più nota Nuova Yorch) e del nome del protagonista, che passa da Giuseppe (ritenuto forse troppo "provinciale") ad Alberto e si guadagna anche la definizione di spiritualista: evidente come l'innalzamento "filosofeggiante" del registro che ne consegue sia funzionale all'effetto dissacrante cui egli è sottoposto. D'altronde l'amplificatio a fini antifrastici è costante nelle scelte stilistiche: una frase semplice e diretta di (b) come se vi sono i medici del corpo diventa in (c), senza alcun aumento di informazione ma con risultato ben diverso, «se le dotte e pietose cure d'una porzione preclarissima dell'umana famiglia si dedicano a guarire i corpi malati dei loro simili», dove si nota, oltre alla tendenza perifrastica, la doppia aggettivazione, l'allitterazione (della $p$ ) e il ricercatissimo superlativo, che contribuiscono all'effetto "perverso" e allo scopo umoristico.

9. La seconda innovazione "strutturale" è l'articolazione narrativa relativa all'esposizione del cartello, che appare come uno sviluppo dell'attesa di sette anni concepita in (b) e assente nel modello francese: in (c) all'esposizione del primo cartello segue infatti un insuccesso che cessa solo quando il medico si risolve a specificare che il pagamento sarà posticipato. Si tratta con tutta evidenza di un rafforzamento della satira antiborghese, cui ancora una volta partecipa coerentemente il piano linguistico, con inserti quali si sfregolò le mani e cure a cottimo, che demistificano qualunque allure di nobiltà dell'attività del medico, la cui definizione di pratico spiritualista è tendenzialmente ossimorica e dunque dissacrante rispetto al solo epiteto precedente; il referenziale celebre dottore di (b) si innalza (con variatio ed ironia) in audace novatore, mentre all'ex-droghiere viene preposto l'aggettivo onesto, quasi a suggerire sottilmente che la sua malattia, più che una patologia personale, sia qualcosa di fisiologicamente connesso al mestiere che ha svolto.

10. A questa complicazione fa invece da contraltare l'eliminazione della contrattazione tra medico e paziente inserita in (b) con scopi non diversi, ma con una trovata forse più banale e allo stesso tempo narrativamente onerosa; (c) è più vicino ad (a) anche nell'espunzione del riferimento ai giornali americani, sviluppo in (b) di quello presente nella brevissima introduzione di (a). La scelta può essere dettata forse dall'intenzione di non calcare troppo la mano sulla localizzazione della storia, e avere quindi finalità "generalizzanti", per non dire che Nievo può aver avvertito eccessiva la citazione di fonti americane nel momento in cui fruiva di quella francese.

11. Una terza aggiunta strutturale in (c) è la necessità della perizia, che prende il posto del lungo dialogo di (a), cui è comunque strutturalmente 
più vicino in questa zona: sembra che qui Nievo voglia mettere a frutto la propria formazione giuridica (e la conoscenza che gli deriva anche dal processo subito a partire dal 1857 per l'Avvocatino) per dettagliare la procedura e utilizzare a scopo ironico il connesso lessico tecnico e gergale (ragioni, obbiezioni, prove, eccezioni, fatto, negativa, pro' e contro). In prossimità della stessa si noti en passant anche la frase inserita a commento del collegio giudicante costituito da 12 membri, «numero poco prediletto dagli studiosi della cabala ${ }^{37}$ : più che per un valore negativo in sé, tuttavia, è probabile che esso non soddisfi Nievo in quanto si scosta dal suo numero preferito, 1'11, ritenuto carico di valori simbolici positivi, cui egli allude più volte nei suoi scritti (seppur sempre in tono scherzoso).

12. La successiva innovazione riavvicina (c) ad (a) per quanto riguarda il numero dei processi, in quanto ripristina la sequenza tra il voto in dissenso dell'ultimo giudice e la nullità del processo stesso, senza far menzione di un secondo giudizio. Significativa anche l'evoluzione del particolare relativo all'assenza del medico come causa addotta per la mancata guarigione dell'anima, che, semplificato in (b) ad un indefinito meeting, rispetto a quello politico di (a), ritrova in (c) una caratterizzazione, ma di natura sportiva, e la sviluppa nelle forme della presa in giro delle pratiche dell'alta società abituale nei pezzi umoristici nieviani, tanto nel merito (la corsa di cavalli) quanto nel linguaggio (con l'uso di forestierismi alla moda come steeplechase, 'gara ippica a ostacoli').

13. La conclusione resta tuttavia ben più ampia in (c) che in (a), ed è costellata da una serie di innovazioni anche rispetto a (b): si dice infatti che, seppur nullo, il processo ha attirato l'attenzione su questa nuova tipologia di terapia, allargandone l'utenza potenziale. La circostanza è utilizzata per fornire un terzo step alle modifiche del cartello, in quanto il dottore elimina il termine posticipato, garantendosi un pagamento immediato e mettendosi così al riparo da altri contenziosi, innovazione che accresce la natura strumentale e materialistica dell'operazione e dunque la satira antiborghese di Nievo. Viene inoltre rafforzata la declinazione a fini interni appena accennata in (b): la voce narrante professa infatti l'intenzione di utilizzare in Europa l'innovazione della cura delle anime, laddove in precedenza questa era considerata solo un'opportunità.

14. Segue quindi un epilogo quantitativamente non esiguo in cui Nievo precisa che adopererà subito la soluzione del pagamento anticipato, giustificandosi autoironicamente con le necessità della propria anima, e inscenando un dialogo fittizio con gli interlocutori, alle cui ipotetiche obiezioni sulla commistione tra spiritualità e materialismo risponde in tono insieme

${ }^{37}$ Anche questo tema è presente nel Barone di Nicastro, il cui stemma «era una bilancia in campo rosso col motto cabalistico: Pensare e pesare» (Nievo 1967: 514). 
apodittico e scherzoso, prima di ribadire che chi dovesse pretendere la restituzione dei denari per mancata guarigione dovrà dimostrare di avere un'anima, cosa tutt'altro che scontata nella contemporaneità: ulteriore tassello della polemica "etico-politica" che anima il suo giornalismo (seppur condotta qui in tono lieve) contro lo spirito inerte del tempo, rinunciatario e privo di midollo.

Caso raro in cui possiamo assistere all'evoluzione in tre stadi del lavoro nieviano, il confronto suggerisce persino che, con qualche approssimazione, essi si potrebbero avvicinare alle tre parti della formazione del discorso classico: l'inventio, qui corrispondente al reperimento dell'aneddoto nella fonte; la sua dispositio in un'articolazione parzialmente mutata dei nuclei narrativi, ma ancora poco elaborata sul piano degli accorgimenti retorici nel primo articolo; e il lavoro di elocutio che (insieme ad alcuni cambiamenti strutturali) ne trasforma il genere adattandolo pienamente al registro ciarliero - si noti il "colore" degli inserimenti di riferimenti colti (Voltaire) o di cronaca (Pagliano) - e alle finalità della zona più elaborata e significativa del giornalismo nieviano, quella dei pezzi umoristico-satirici di $U P$, che devono rispondere insieme a diverse esigenze, e unire all'intrattenimento asciuttamente aneddotico, magari spazialmente diluito, una finalità più chiaramente satirico-moralistica, con allusioni filosofiche (al materialismo), culturali (antiamericane) e politiche (sull'Europa). Un caso limite, certo, ma anche una circostanza che rafforza il ruolo non generico della lingua e della cultura francese nei testi nieviani, getta nuova luce sulle modalità compositive dei suoi testi (almeno giornalistici) e invita ad ulteriori indagini su questo fronte.

\section{BIBLIOGRAFIA}

Bacot, J.-P. (2005). La presse illustrée au XIX'e siècle. Une histoire oubliée. Limoges: Presse universitaire de Limoges.

Chaarani Lesourd, E. (2002). A la folie!... Pas du tout? - Je t'aime. Portrait(s) d'Ippolito Nievo et de Matilde Ferrari. In A. Morini (a cura di), Figure, Figures, Portraits d'hommes et de femmes célèbres, ou moins, dans la littérature italienne (pp. 51-91). St. Etienne: Université de St. Etienne.

Cutter, W. R. (a cura di). (1908). Genealogical and personal memoirs. Relating to the Families of Boston and Eastern Massachusetts. Vol. 1. New York: Lewis Historicale Publishing Company [rist. Baltimore: Clearfield, 1995, 2000, 2008).

Garau, S. (2013). «La morbida Rivista de’ due mondi». Nievo lettore della «Revue des deux Mondes». In E. Del Tedesco (a cura di), Ippolito 
Nievo centocinquantanni dopo, Atti del Convegno, Padova, 19-21 ottobre 2011 (pp. 315-330). Pisa-Roma: Serra.

Gorra, M. (1985). Il diavolo nella biblioteca di Nievo, ossia Le Sage e De Vigny, Belfagor, 3, [30 settembre], 577-588.

Heine, H. (1855a). Poëmes et Légendes par Henri Heine. Paris: Calmann Lévy.

Heine, H. (1855b). De l'Allemagne par Henri Heine, nouvelle édition entièrement revue et considérablement augmentée. Paris: Michel Lévy Frères.

Mengaldo, P. V. (1987). L'epistolario di Nievo. Un'analisi linguistica. Bologna: il Mulino.

Motta, A. (2018). Su un articolo del «Corriere delle Dame» da attribuirsi a Nievo, la sua fonte francese e altre tre varietà. Lettere Italiane, 1, $80-99$.

Nievo, I. (1964). Quaderno di traduzioni, a cura di I. De Luca. Torino: Einaudi.

Nievo, I. (1967). Tutte le opere narrative, a cura di F. Portinari. Milano: Mursia.

Nievo, I. (1981). Lettere, a cura di M. Gorra. Milano: Mondadori.

Nievo, I. (2008). Scritti giornalistici alle lettrici, a cura di P. Zambon. Lanciano: Carabba.

Nievo, I. (2015). Scritti politici e d'attualità, a cura di A. Motta. Venezia: Marsilio.

Samaritani F. \& Zambon, P. (2002). Nota nieviana: la biblioteca di casa Nievo. Archivi del Nuovo. Notizie di casa Moretti, 10-11, 55-68.

Strenna (1859). Un pensiero a Venezia. Strenna pel 1860 dedicata alle donne italiane. Edizione a beneficio dell'emigrazione veneta. [Milano]: Canadelli e Compagno.

Tommaseo, N. \& Bellini, B. (1861-1879). Dizionario della lingua italia$n a$, consultabile on-line all'URL: http://www.tommaseobellini.it/\#/ [31/05/2018].

Vreto, M. (1855). Contes et Poèmes de la Grèce moderne, précédés d'une introduction par P. Mérimée de l'Académie française. Paris: Audois.

\section{TRANSLATIONS AND REWRITING: HOW NIEVO (JOURNALIST) WORKED}

\section{Summary}

For Nievo, the French language and French magazines represented the mediation of European cultural models as well as a vehicle for textual sources, which the journalist rewrote, pushing translation to its limits. By attributing to Nievo an anonymous article, 
appearing in 1859 in the italian magazine Corriere delle Dame, thanks to the comparison with another article he wrote for L'Uomo di Pietra, and tracing its French source to an article published in Le Monde Illustré, we are provided with a unique opportunity to compare the three texts and to gain insights into the modus operandi of this man of letters.

Keywords: Nievo as Journalist, French as a vehicular Language, $19^{\text {th }}$ Century Magazines, Rewriting and Translation, Text Analysis, the Italian Risorgimento. 